\section{Transitions in infant learning are modulated by dopamine in the amygdala}

\author{
Gordon A Barr ${ }^{1,2}$, Stephanie Moriceau ${ }^{3-5}$, Kiseko Shionoya ${ }^{3}$, \\ Kyle Muzny ${ }^{3}$, Puhong $\mathrm{Gao}^{2}$, Shaoning Wang ${ }^{1,2} \&$ \\ Regina M Sullivan ${ }^{3-5}$
}

Behavioral transitions characterize development. Young infant rats paradoxically prefer odors that are paired with shock, but older pups learn aversions. This transition is amygdala and corticosterone dependent. Using microarrays and microdialysis, we found downregulated dopaminergic presynaptic function in the amygdala with preference learning. Corticosterone-injected 8-d-old pups and untreated 12-d-old pups learned aversions and had dopaminergic upregulation in the amygdala. Dopamine injection into the amygdala changed preferences to aversions, whereas dopamine antagonism reinstated preference learning.

Transitional phases in development are associated with specific behaviors in specific social contexts. For example, infants of many altricial species, including birds, dogs and primates, learn attachments to their caregivers despite diverse environments that include both positive and noxious stimulation ${ }^{1,2}$. These early conditioned preferences promote attachment to the mother and encourage infants to remain in proximity to her, regardless of the quality of care (Supplementary Table 1). This can be considered to be a prerequisite for both attachment, which consists of enhanced approach and attenuated avoidance, and safety learning in that the learned cues, both positive and negative, signal the safety of the dam and nest ${ }^{2}$. However, with maturation, the infant transitions out of the specialized attachment learning period. Because

Figure 1 Role of shock on corticosterone and behavior. (a) At $8 \mathrm{~d}$ of age, pups are in the stress hypo-responsive period ${ }^{15}$, in which mild shock $(0.5 \mathrm{~mA})$ did not elevate mean plasma corticosterone (cort) levels. In contrast, injection of corticosterone in a saline vehicle (at $24 \mathrm{~h}$ and $30 \mathrm{~min}$ before conditioning; $3.0 \mathrm{mg}$ per $\mathrm{kg}$ of body weight, intraperitoneal) produced elevated plasma corticosterone levels following shock, roughly equal to that induced by mild shock at $12 \mathrm{~d}$ of age (ANOVA, significant group effect, $F_{3,9}=9.95, P<0.005$ ). (b) Infant odor-shock conditioning paradoxically caused an odor preference in 8-d-old pups, although similar conditioning in 12-d-old pups caused an odor aversion. This odor aversion can be learned in 8-d-old rats if injected with corticosterone before conditioning. Control groups (unpaired odor-shock and odor-only pups) failed to learn either an aversion or preference, regardless of the age or corticosterone treatment. The dotted line represents chance performance (ANOVA, significant interaction between conditioning groups and drug treatment, $F_{4,49}=16.07, P<0.0001$ ). Error bars represent mean \pm s.e.m. Blue indicates conditions in which preferences are learned and red indicates conditions in which aversions are learned.

our understanding of the mechanisms that control developmental transitions is limited, we used an attachment/fear transition model system to explore the amygdala neural changes underlying this transition.

To understand the mechanisms in the amygdala that mediate early preference learning and the corticosterone-induced switch to aversion learning, we used an olfactory odor-shock learning procedure to condition rat pups to learn an odor preference at $8 \mathrm{~d}$ of age (postnatal day $8, \mathrm{P} 8)$ or an odor aversion at the same age when treated with corticosterone. We also tested 12-d-old rats (P12) that normally learn an aversion. There was an endogenous increase in shock-induced corticosterone that was essential for avoidance learning and induced the switch from odor preference to avoidance learning irrespective of age (Fig. 1a). Between these ages (at $10 \mathrm{~d}$ of age), pups transitioned from odor-shock preference learning to more adult-like, amygdaladependent fear/odor-avoidance learning (Fig. 1b). The amygdala, which is rich in glucocorticoid receptors ${ }^{3}$, only participated in conditioning during aversion learning, again regardless of age, but dependent on corticosterone levels ${ }^{4-6}$.

For broad screening of possible phenotypic-related changes in the amygdala, we used microarrays (Affymetrix) to examine changes in gene expression. We determined differential gene expression using ranked products ${ }^{7}$ followed by confirmatory quantitative PCR analyses (see Table 1 for $P$ values; Supplementary Methods and Supplementary Tables 2-6). One class of genes that related to presynaptic dopamine function followed the behavioral phenotype and consisted of tyrosine hydroxylase $(T h)$, the dopamine transporter (Slc6a3, also known as DAT), dopa decarboxylase $(D d c)$ and the solute carrier 18a2 (Slc18a2, also known as VAT2, which loads monoamines into vesicles). An aldehyde dehydrogenase (Aldh1a1), which is highly and specifically expressed in dopaminergic cells of the substantia nigra and ventral tegmental area $^{8}$, also followed the phenotype. At P8, each of these dopaminergic components was significantly downregulated
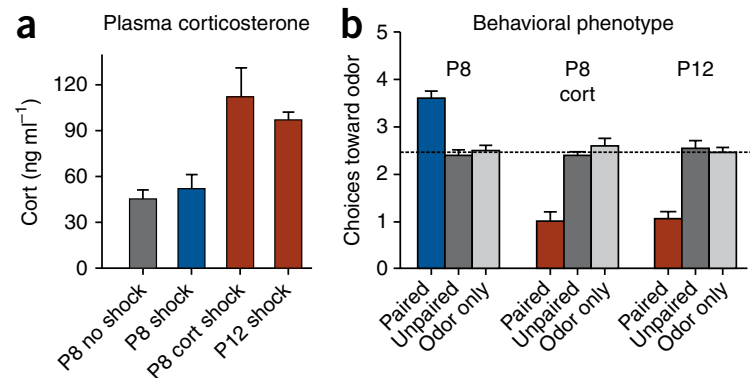

\footnotetext{
${ }^{1}$ Department of Anesthesiology and Critical Care Medicine, Children's Hospital of Philadelphia, Philadelphia, Pennsylvania, USA. ${ }^{2}$ Department of Developmental Neuroscience, New York State Psychiatric Institute, New York, New York, USA. ${ }^{3}$ Department of Zoology, University of Oklahoma, Norman, Oklahoma, USA. ${ }^{4}$ Emotional Brain Institute, Nathan Kline Institute, Orangeburg, New York, USA. ${ }^{5}$ Child and Adolescent Psychiatry, New York University Langone Medical Center, New York, New York, USA. Correspondence should be addressed to G.A.B. (barrg@email.chop.edu) or R.M.S. (regina.sullivan@nyumc.org).
} 
Table 1 Microarray and PCR results

\begin{tabular}{|c|c|c|c|c|c|c|c|c|c|c|c|}
\hline \multicolumn{5}{|c|}{$8 \mathrm{~d}$ old } & \multicolumn{4}{|c|}{$8 \mathrm{~d}$ old, corticosterone treated } & \multicolumn{3}{|c|}{$12 \mathrm{~d}$ old } \\
\hline Gene & $P$ & Array fold & PCR fold & Direction & $P$ & Array fold & PCR fold & Direction & $P$ & Array fold & Direction \\
\hline Aldhlal & 0.000 & -2.03 & -1.64 & Down & 0.584 & 1.30 & 2.21 & Up & 0.025 & 1.77 & Up \\
\hline$D d c$ & 0.004 & -1.95 & & Down & 0.782 & -1.23 & & & 0.846 & 1.18 & \\
\hline Drdla & 0.020 & 2.12 & & Up & 0.212 & -1.72 & & & 0.213 & -1.40 & \\
\hline Drd2 & 0.192 & 1.44 & & Up & 0.417 & -1.29 & & & 0.408 & -1.33 & \\
\hline SIc18a2 & 0.001 & -2.26 & & Down & 0.564 & 1.24 & & & 0.070 & 1.80 & Up \\
\hline Slc6a3 & 0.000 & -3.49 & -4.97 & Down & 0.052 & 2.14 & 2.27 & Up & 0.016 & 2.25 & Up \\
\hline Th & 0.000 & -3.32 & -2.92 & Down & 0.003 & 1.89 & 1.82 & Up & 0.071 & 1.30 & Up \\
\hline
\end{tabular}

Comparisons are between pups for which odor was paired with shock and those for which odor and shock were unpaired. The $\mathrm{P}$ values were corrected for multiple testing7. Fold change was calculated as the ratio of the paired to unpaired conditions. For the PCR data, fold change is by the delta-delta Ct method using Gadph as a control

(Supplementary Methods). All rats were killed immediately after conditioning. For P8 paired pups, all of the presynaptic markers were downregulated (blue) and both Drd1a and Drd2 were upregulated (red). Aspects of other neurotransmitters changed, but not consistently with the phenotype (Supplementary Tables 2-4). At 12 d, the pattern was reversed, although not all of the probe sets were significant. Likewise, and consistent with the behavioral data, corticosterone treatment changed the pattern of gene expression from that of a P8 pup to that of a P12 pup when conditioned (again, not always significantly). Fewer pups were used for the P12 untreated $(n=4)$ and P8 corticosterone-treated ( $n=3$ ) groups than for the normal P8 group $(n=7)$. This might account for the fewer significant effects. The University of Oklahoma Institutional Animal Care and Use Committee approved all of the experiments. Data were deposited in GEO (accession code GSE17651).

in the odor-shock paired group compared with the controls (Table 1). Conversely, pups learning an odor aversion (P8 corticosterone paired and $\mathrm{P} 12$ paired) showed significant upregulation of a subset of these dopamine-related genes. At $12 \mathrm{~d}$ of age, four dopamine-related genes were significantly upregulated (Th, Slc6a3, Slc18a2 and Aldh1a1; Table 1). Notably, pretreatment of P8 pups with corticosterone resulted in upregulation of Th and Slc6a3, similar to the P12 pups. Dopamine receptors ( $D r d 1 a$ and $D r d 2$ ) were regulated in a phenotypic direction, but opposite to that of the presynaptic markers. Other genes for other transmitter systems were up- or downregulated by learning, but not in a phenotypically specific manner (Supplementary Tables 2-4).

We then directly assessed amygdala dopamine efflux during odorshock conditioning in 8-d-old pups using in vivo microdialysis with high-performance liquid chromatography. Conditions that allow preference learning (without corticosterone) decreased dopamine efflux from the amygdala (Fig. 2a), whereas conditions that favor aversion learning (with corticosterone) increased amygdala levels of dopamine (Fig. 2b). Pups receiving unpaired presentations showed less change in dopamine efflux than did paired pups $(P>0.05)$, but in the same direction (see Supplementary Figs. 1 and 2 for metabolites, and Supplementary Fig. 3 for probe placements). We did not

Figure 2 Dopamine efflux and manipulation. (a) Measurements of extracellular dopamine efflux in the amygdala at P8 before (baseline), during (conditioning) and after (recovery) conditioning. Paired odor-shock treatment, which normally produces an odor preference, decreased amygdala dopamine, but increased amygdala dopamine in $\mathrm{P} 8$ rats that normally learn an aversion after injection with corticosterone (data collapsed over 45-min bins for presentation, significant interaction between conditioning groups $\times$ time, $F_{18,135}=88.81$, $P<0.0001$ ). (b) P8 pups learning an aversion (with corticosterone) had increased amygdala levels of dopamine (significant interaction between conditioning groups $\times$ time, $F_{18,135}=71.87, P<0.0001$ ). Post hoc Fisher tests revealed that all paired groups were significantly different from the control groups $(P<0.001)$ during conditioning for both saline and corticosteronetreated groups. (c) At $8 \mathrm{~d}$ of age, dopamine infused (3-6 $\mu \mathrm{g}$ per $2 \mu \mathrm{l}$ at $0.1 \mu \mathrm{l}$ per min, beginning $5 \mathrm{~min}$ before conditioning) into the amygdala resulted in a learned odor aversion during an odor-shock conditioning. Saline-infused rats of the same age learned the expected preference for the odor (condition $\times$ infusion interaction; $F_{1,17}=37.36, P<0.0001$ ). (d) Blocking amygdala dopamine receptors with the receptor antagonist cis-flupenthixol (20 $\mu \mathrm{g}$ per $2 \mu \mathrm{l}$, as per dopamine) caused P8 pups receiving corticosterone to switch to an odor preference rather than the odor aversion seen with corticosterone injection during an odor-shock conditioning in rats of the same age (interaction between conditioning groups, drugs infusion and corticosterone/saline, $F_{1,32}=17.79$, $P<0.0005)$. Post hoc Fisher tests showed that paired groups differed from controls for both dopamine and cis-flupenthixol experiments. obtain similar phenotypically consistent results with norepinephrine or serotonin efflux in the same samples (data not shown). The results are consistent with previous studies on fear and safety learning and the role of dopamine in the amygdala ${ }^{9-11}$.

The microdialysis experiments confirmed our microarray data and suggest that low levels of dopamine in the amygdala assist in preventing odor aversion learning in rat pups at young ages. To test this more directly, we implanted pups with bilateral amygdala cannulas at $6 \mathrm{~d}$ of age and returned them to the nest (see Supplementary Fig. 4 for cannula placements $)^{5,6}$. At $8 \mathrm{~d}$ of age, pups were infused with saline, dopamine or a dopamine receptor antagonist (cis-flupenthixol) during odor-shock conditioning and returned to the nest. The next day, a Y-maze test assessed odor-preference/aversion learning. Infusions of dopamine into 8-d-old pups caused the age-typical odor-preference learning to switch to aversion learning (Fig. 2c). In contrast, blocking amygdala dopamine receptors in pups treated with corticosterone resulted in preference learning even though those receptors were upregulated at this age (Table $\mathbf{1}$ and Fig. 2d).

Infants are uniquely adapted to regulate transitions during development to insure that different age-appropriate 'safe' behavioral strategies are engaged. Experimental data and clinical experience suggest

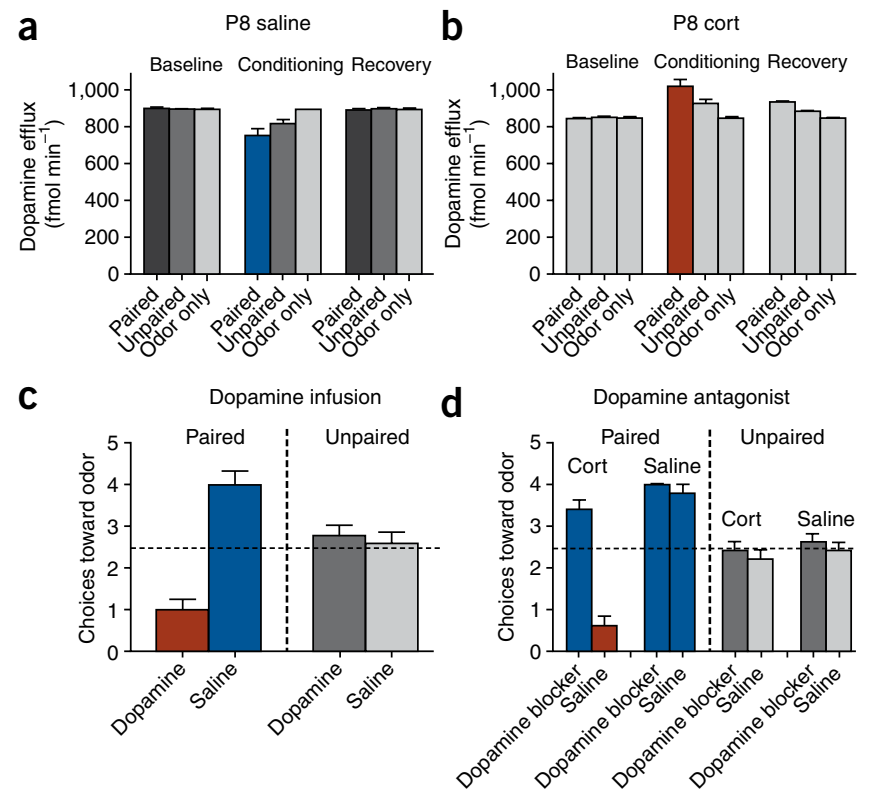




\section{BRIEF COMMUNICATIONS}

that attachment by the altricial infant to the caretaker has evolved to ensure that the infant forms a bond to that caregiver regardless of the quality of care received. Downregulation of the presynaptic elements of dopamine transmission in the amygdala may be one neural mechanism, at least in the rat, by which the amygdala is prevented from participating in avoidance/fear conditioning during attachment learning to the maternal odor. Notably, the transition out of this specialized period of preference learning did not depend on the pups' age (within limits), but rather on corticosterone levels that are normally regulated by the mother. Thus, dopaminergic activity 'flips' from inhibition to activation in a circuit that includes at least the amygdala, which is fully consistent with phenotype rather than age, and is probably responsive to levels of stress. The amygdala and the ventral tegmental dopaminergic cells contain glucocorticoid receptors ${ }^{3,12}$ and corticosterone enhances the firing rates of dopaminergic cells ${ }^{13}$. Rapid switches are crucial to survival for both adults and infants, and the mechanisms required to react with age-appropriate behaviors to similar stimuli in different contexts (for example, stress) may predestine similar mechanisms in the adult ${ }^{14}$.

Our results closely parallel the role of dopamine in amygdaladependent fear conditioning in the adult, where increased activation of dopaminergic neurons in the amygdala modulates fear-related behaviors ${ }^{9,10}$. Moreover, there are parallels between dopaminergic mechanisms in the amygdala that mediate the preference learning shown here and safety learning in the adult ${ }^{10,11}$ : dopamine function was decreased in the amygdala, dopamine agonists decreased preference learning and abolished the safety response, and dopamine antagonists abolished aversion learning and enhanced the safety response. Thus, processes that mediate preference learning in the young pup, and that serve to keep the pup safely in the nest and to foster attachment to the dam, may also signal safety in the adult.

Note: Supplementary information is available on the Nature Neuroscience website.

\section{ACKNOWLEDGMENTS}

We thank L. Levita and the anonymous reviewers for helpful criticisms. This work was supported by grants NIH-NICHD-HD33402, NSF-IOB-0544406, NIH-NIDCD-DC009910 and the Leon Levy Foundation to R.M.S. and NIH-NIDA DA00325 and NIH-NIMH MH080603 to G.A.B.

\section{AUTHOR CONTRIBUTIONS}

G.A.B. helped conceptualize and design the project, designed the microarray experiments, helped analyze the array and PCR data, and wrote the manuscript. S.M. helped conduct the behavioral, pharmacological studies, analyzed the data, and contributed to writing the manuscript. K.S. conducted the highperformance liquid chromatography studies. K.M. helped collect data for many of the experiments. P.G. analyzed the microarray and PCR data. S.W. conducted the microarray and PCR studies and carried out preliminary data analysis. R.M.S. helped conceptualize and design the project, supervised the behavioral, pharmacological, and dialysis studies, and helped write the manuscript.

Published online at http://www.nature.com/natureneuroscience/.

Reprints and permissions information is available online at http://www.nature.com/ reprintsandpermissions/.

1. Maestripieri, D., Lindell, S.G., Ayala, A., Gold, P.W. \& Higley, J.D. Neurosci. Biobehav. Rev. 29, 51-57 (2005).

2. Sullivan, R.M., Moriceau, S., Raineki, C. \& Roth, T.L. in Cognitive Neuroscience IV (ed. M. Gazzaniga) 889-904 (MIT Press, Cambridge, Massachusetts, 2009).

3. Meaney, M.J., Sapolsky, R.M. \& McEwen, B.S. Brain Res. 350, 159-164 (1985).

4. Sullivan, R.M., Landers, M., Yeaman, B. \& Wilson, D.A. Nature 407, 38-39 (2000).

5. Moriceau, S. \& Sullivan, R.M. Nat. Neurosci. 9, 1004-1006 (2006).

6. Moriceau, S., Wilson, D.A., Levine, S. \& Sullivan, R.M. J. Neurosci. 26, 6737-6748 (2006).

7. Breitling, R., Armengaud, P., Amtmann, A. \& Herzyk, P. FEBS Lett. 573, 83-92 (2004).

8. Galter, D., Buervenich, S., Carmine, A., Anvret, M. \& Olson, L. Neurobiol. Dis. 14 637-647 (2003).

9. Rosenkranz, J.A. \& Grace, A.A. Nature 417, 282-287 (2002).

10. Bissière, S., Humeau, Y. \& Luthi, A. Nat. Neurosci. 6, 587-592 (2003).

11. Pollak, D.D. et al. Neuron 60, 149-161 (2008).

12. Härfstrand, A. et al. Proc. Natl. Acad. Sci. USA 83, 9779-9783 (1986).

13. Overton, P.G., Tong, Z.Y., Brain, P.F. \& Clark, D. Brain Res. 737, 146-154 (1996).

14. Herry, C. et al. Nature 454, 600-606 (2008).

15. Levine, S., Dent, G. \& De Kloet, E.R. in Encyclopedia of Stress, Vol. 3 (ed. G. Fink) 518-526 (Academic Press, New York, 2000). 